\title{
Research on Global Energy Optimization Allocation Mechanism in the Background of Global Energy Internet
}

\author{
Ming Zeng ${ }^{1}$, Tong Yan ${ }^{1}$ a, Zhuhan Long ${ }^{1}$ Liying Wang $^{1}$ and Chenjun Sun ${ }^{2}$ \\ ${ }^{1}$ North China Electric Power University, Beijing 102206, China. \\ 2 State Grid Hebei Electric Power Company, Hebei 050000, China. \\ ayantong1208@126.com
}

\begin{abstract}
The Global Energy Internet aims to build a new global energy optimal configuration that focuses on clean energy and electricity. This paper studies the global energy configuration mechanism in the context of the Global Energy Internet. First, qualitatively analyze the Global Energy Internet's contribution to the optimization of global energy configuration from the perspectives of energy supply costs and networking benefits. Second, a global energy optimal configuration model with the goal of minimizing energy distribution costs has been established. The differences in the global energy resources optimization and allocation scheme under the traditional mode and the Global Energy Internet mode are comparatively analyzed through examples to quantitatively elaborate the impact of the Global Energy Internet on the global energy optimal configuration.
\end{abstract}

Keywords: energy optimal configuration; Global Energy Internet; energy distribution cost.

\section{Introduction}

At present, global energy resources and energy consumption are unevenly distributed. Energy development is increasingly concentrated in a few countries and regions. Some countries with few resources have continued to increase their dependence on energy, and energy security issues are severe. In order to meet the energy needs of various countries, large-scale energy allocation needs to be optimized globally. Traditional fossil fuels are transported through oil tankers, oil/gas pipelines, and railways/roads, which takes a long time, is costly, and has serious pollution. As a result, the allocation of energy resources centered on electric energy has gradually gained attention with the advantages of rapidity, convenience, economy, and environmental protection. However, at this stage, the world's power distribution is limited in scale. Compared with fossil energy, the global power trade scale is very small, which is equivalent to about 80 million tons of standard coal, less than $2 \%$ of fossil energy trade volume, and cannot meet the requirements of future energy clean development; The world's existing electric power deployment has a limited range, and the economical transmission distance of existing ultra-high voltage power grids is generally 500 to 1000 kilometers, which cannot meet the needs of future large-scale development and deployment of energy sources. Therefore, it is urgent to establish a global energy distribution network platform with electricity as the center, a higher voltage level, greater transmission capacity, and more long distances. The Global Energy Internet has emerged as a result [1-2].

The Global Energy Internet is based on the UHV grid as the backbone of the network (passage), to promote the transmission of clean energy as the dominant global interconnected strong smart grid. The Global Energy Internet will consist of multinational cross-border backbone grids and ubiquitous smart grids covering all voltage-grade power grids (transmission grids, distribution grids) of various countries, linking "one pole and one trail” with large-scale energy bases in various states to adapt to various types of distribution. Power access needs, able to deliver wind energy, solar energy, ocean energy and other renewable energy to various users, is a global energy configuration platform with a wide range of services, strong configuration capabilities, high safety and reliability, and green and low carbon [3].

At present, the research on the optimal configuration of energy is mostly limited to the national level. By constructing fuzzy multi-objective multi-exponential transport model, literature [4] considers the external cost of energy as well as time cost, loss and investment, and establishes the best energy transportation system in China. The literature [5] aims at maximizing the green economic 
benefits of energy consumption, using the principle of equalization of marginal benefits of energy consumption, and the theory of marginal utility in economics to establish a model for the optimal deployment of renewable energy to study the optimal configuration of renewable energy in China. However, the existing research on the energy configuration of the Global Energy Internet is limited to qualitative analysis. For this reason, this paper analyzes the global energy optimal configuration in the context of the Global Energy Internet and analyzes the contribution of the Global Energy Internet to the realization of global energy optimization from qualitative and quantitative perspectives. On the one hand, from the two aspects of energy supply cost and networking benefits, we will qualitatively analyze the role of Global Energy Internet in promoting global energy optimal configuration. On the other hand, a global energy optimal configuration model with the goal of minimizing energy distribution costs has been established. The differences in the global energy resources optimization allocation scheme under the traditional mode and the Global Energy Internet mode have been comparatively analyzed through examples and the impact of the Global Energy Internet on global energy optimization has been illustrated.

\section{Global Energy Internet Achieves Global Energy Optimal Configuration}

The global grid interconnection can achieve the optimal configuration of global energy from two aspects: reducing energy supply costs and obtaining networking benefits:

(1) Reduce energy supply costs

Relying on the Global Energy Internet, we have achieved large-scale development of clean energy and benefits from outsourcing, which can effectively reduce the cost of electricity supply. Taking Asia-European transmission as an example, send clean energy such as natural gas, wind energy and solar energy to the sending end regions in Asia (China's Xinjiang, Kazakhstan, Russia's Siberia, and Mongolia) and transmitting electricity to Germany using $\pm 1100 \mathrm{kV}$ UHVDC transmission technology. The number of DC channel utilization hours is 5,500 hours. Compared with Germany's receiving offshore wind power, the Asia and Europe intercontinental power transmission project through Russia's St. Petersburg relay transmission scheme is $30.4 \%$ cheaper than German offshore wind power, and the maximum power price difference is 0.3648 yuan $/ \mathrm{kWh}$. In the Asia-Europe direct transmission scheme, the price difference is the largest It is 0.526 yuan $/ \mathrm{kWh}, 43.8 \%$ cheaper than German offshore wind power. By implementing Asia-Europe transmission, the cost of electricity supply in Germany can be effectively reduced, and the benefits of intercontinental transmission are significant.

(2) Get significant networking benefits

Because of the time difference between the continents, the establishment of global energy-based networking, and the interconnection of the power grids on all continents, it is possible to effectively use the complementarity of the power load characteristic curves of all continents, to conduct interstate peak-valley adjustments, and to optimize the deployment of renewable energy resources on a global scale ,to improve the utilization of power generation equipment on all continents and reduce system spare capacity. Take the three continents of the northern hemisphere, Europe, North America and Asia, for example in 2050. The North American power grid spans the West 4th District to the West 10th District, and the Northeast Asian Power Grid spans the East 7th District to the East 9th District. The European Synchronous Internet spans the 0 time zone to the East 2 zone. After global networking, the natural time difference of each state can be used to optimize the global grid load and form a relatively smooth load curve to realize the peak load shifting effect. Figure 1 compares the load curves before the three regional networks and the fitted load curves after networking. It can be seen that under the condition that the power grids in each state are fully interconnected, the effect of complementarity between peaks and valleys in the three major regional networks is obvious. After the network is connected, the load distribution in each period of the day will be balanced and the peak-to-valley load difference is reduced from $25 \%$ to $40 \%$ of the three regional grids to less than $10 \%$. 


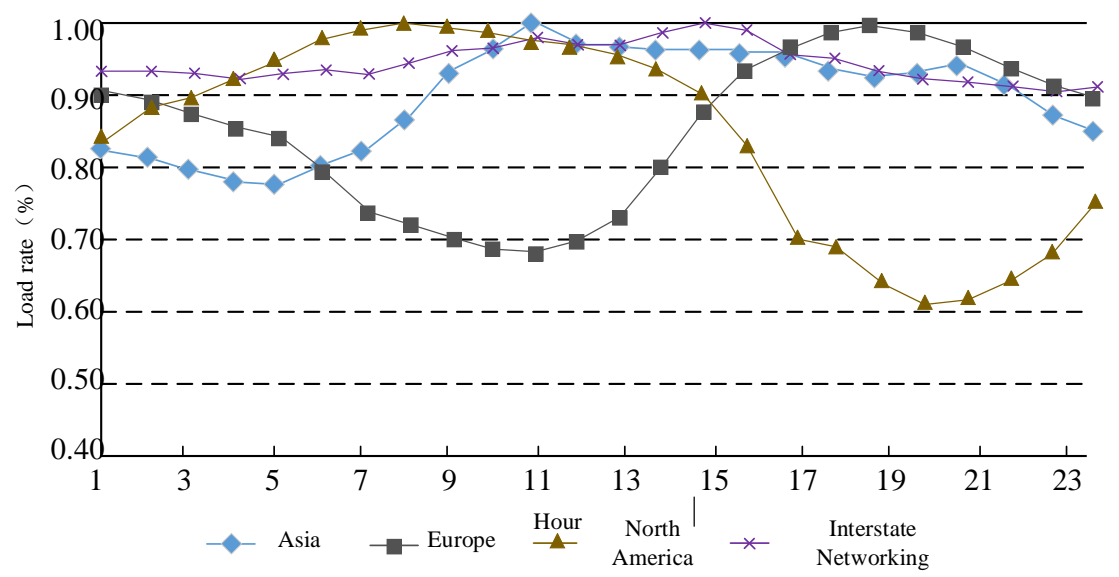

Fig.1 Illustration of the Complementary Relationship among Load Curves in Europe, North America and Asia

\section{The Global Energy Optimal Configuration Model under the Background of Global Energy Internet}

Energy transmission is the inevitable result of the imbalance of energy distribution. The proper choice of energy transmission mode and energy transmission path is very important for achieving optimal energy configuration. In the process of energy configuration, transmission paths and transmission modes are mainly affected by many factors such as economy, transportation equipment, and energy supply and demand patterns. From an economic point of view, the energy demand center will obtain energy from the energy supply center, which will inevitably generate corresponding costs. Therefore, the cost of energy distribution is used as a quantitative indicator of economic performance. Energy transfer systems such as roads, railways, maritime transport, and power grids limit the maximum and minimum transmission capacity. The energy supply and demand pattern mainly refers to the energy supply and demand in a certain area. The amount of energy available is the maximum amount of energy that can be exported to the energy output area. The amount of energy that can be transferred to an area should not exceed this value. Energy demand refers to the amount of energy required to enter the area. The amount of energy that is transmitted within a certain area should not be exceeded, in order to meet the energy needs of the area. Therefore, this section establishes a global energy optimal configuration model based on the analysis of the above-mentioned global energy allocation influencing factors, and compares and analyzes the differences between the global energy resources optimal configuration scheme under the traditional mode and the Global Energy Internet mode in combination with the calculation examples, so as to quantitatively describe the impact of the Global Energy Internet on global energy optimal configuration.

\subsection{Section Headings.}

(1) Objective Function

The optimization of the global energy configuration is based on the optimization of economic indicators, that is, to minimize the energy distribution cost objective function.

$$
\min \sum_{k} a_{k}(i, j) \cdot x_{k}(i, j)
$$

In the formula, $\mathrm{i}$ and $\mathrm{j}$ are the energy output area and the energy input area number respectively; $a_{k}(i, j)$ represents the unit cost of the k type of energy; $x_{k}(i, j)$ represents the energy transmission volume of the k energy in the energy output area $i$ and the energy input zone $j$.

(2) Constraint Condition

1) Transportation system capacity constraints

$$
a_{k, \min }(i, j) \leq a_{k}(i, j) \leq a_{k, \max }(i, j)
$$


In the formula, $a_{k, \min }(i, j)$ and $a_{k, \max }(i, j)$ are the minimum and maximum energy transfer energy constraints for the transmission of the k energy from the i energy output area to the $j$ energy input area.

2) Energy supply constraints

$$
\sum_{j} a_{k}(i, j) \leq p(i)
$$

In the formula, the $p(i)$ is the largest energy supply in the i energy output area.

3) Energy demand constraints

$$
\sum_{i} a_{k}(i, j) \geq q(j)
$$

In the formula, $q(j)$ is the minimum energy demand for the $\mathrm{j}$ energy input area.

\subsection{Analysis of examples}

The example divides the world into seven regions, including the Asia Pacific region, the Middle East region, North America, Central and South America, Europe and Eurasia, Africa, and the Arctic, respectively. A comparative analysis of the global energy optimal configuration under the traditional mode and the Global Energy Internet mode. The mathematical model proposed in this paper is established and solved in the generic algebra modeling system GAMS.

(1) Global Energy Optimal Configuration under the Traditional Mode

Based on the transregional transmission of global fossil energy (crude oil and natural gas) in 2010[6], the energy demand and supply in each region are calculated. The calculation results are shown in Table 1 . It is assumed that the maximum capacity of the transmission system is twice as much as the actual transmission capacity of intercontinental energy in 2010.

Table 1. Fossil Energy Demand and Supply in Various Regions under the Traditional Mode

\begin{tabular}{ccc}
\hline region & Demand/million tons of oil equivalent & Supply/million tons of oil equivalent \\
\hline Asia-Pacific & 1047 & 0 \\
the Middle East & 0 & 1000 \\
North America & 331 & 20 \\
Central and South America & 0 & 159 \\
Europe and Eurasia & 309 & 100 \\
Africa & 0 & 408 \\
Arctic region & 0 & 0 \\
\hline
\end{tabular}

According to the model proposed in this paper, the results obtained are that the required energy distribution cost under the traditional mode is 573,551,100 million yuan.

(2) Global Energy Optimal Configuration under the Global Energy Internet Mode

In order to verify the algorithm and simplification issues, assuming that the total global energy demand and total supply remain unchanged, set the power to account for $25 \%$ of the total energy, and the proportion of traditional transmission methods should be reduced accordingly. Calculate crossregional energy demand and supply in each region, the results are shown in Table 2.

Table 2. Energy demand and supply in various regions under the Global Energy Internet mode

\begin{tabular}{ccc}
\hline region & Demand/million tons of oil equivalent & Supply/million tons of oil equivalent \\
\hline Asia-Pacific & 955 & 0 \\
the Middle East & 0 & 863 \\
North America & 331 & 49 \\
Central and South & 34 & 153 \\
America & 367 & 131 \\
Europe and Eurasia & 0 & 396 \\
Africa & 0 & 94 \\
Arctic region & & \\
\hline
\end{tabular}


Assume that the net power price is composed of three parts: on-grid price, line loss power price, and transmission power price of the sending-end power base; among them, the feed-in PV on-grid price is 0.80 yuan $/ \mathrm{kWh}$, and the feed-side wind power on-grid price is $0.47 \mathrm{yuan} / \mathrm{kWh}$. The hydropower at the sending end is $0.30 \mathrm{yuan} / \mathrm{kWh}$, and the transmission power price and the line loss power price vary with the transmission distance. The transmission constraint of the power grid refers to the inter-continental interconnection grid power transmission volume in 2050[7]. In the context of the Global Energy Internet, the global energy optimization configuration results in an energy distribution cost of 53233.59 billion yuan.

\section{Conclusion}

This paper aims at the global energy optimal configuration problem under the background of Global Energy Internet. On the one hand, it qualitatively analyzes the Global Energy Internet's contribution to global energy optimal configuration from two aspects: energy supply costs and networking benefits. On the other hand, a global energy optimal configuration model with the goal of minimizing energy distribution costs has been established. In combination with examples, the differences in the global energy resources optimization allocation scheme under the traditional mode and the global energy internet mode are compared and the impact of the Global Energy Internet on the global energy optimal configuration is described. The results show that the Global Energy Internet has changed the pattern of energy supply and demand and is conducive to reducing energy transmission costs.

The comprehensive utilization cost of energy is also affected by a series of factors such as conversion costs and government policy guidance. In the future, comprehensive evaluation of the comprehensive energy transmission system needs further research.

\section{Acknowledgments}

This work is supported by the Science and Technology Project of State Grid of China (The Impact of Global Energy Internet on the World Political Structure) and the Research Base Program Supported by Beijing Social Science Research Funding (15JDJGA089).

\section{References}

[1]. Liu Zhenya. Global Energy Internet: Must Focus on Electricity [J]. Electric Age. Vol. 40 (2016) No. 1, p. 34-37.

[2]. Wang Yimin. Global Energy Internet Concept and Prospects [J]. China Power. Vol. 49 (2016) No. 3, p. 1-5.

[3]. Liu Zhenya. Global Energy Internet [M]. Beijing: China Electric Power Press, 2015.

[4]. LIN Boqiang, YAO Xin. Power industry location optimization and integrative energy transportation system [J]. Economic Research Journal. Vol. 44 (2009) No. 6, p. 105-115.

[5]. LIU Xiaoli, ZHANG Zezhong, HUANG Qiang, et al. Study on the optimal configuration model of renewable energy facing renewable portfolio standard [J]. Acta Energiae Solaris Sinica. Vol. 24 (2008) No. 2, p. 256-260.

[6]. BP Group. BP World Energy Statistical Yearbook (2011 edition) [R]. London: BP Group, 2011.

[7]. State Grid Energy Research Institute. International Energy and Electricity Price Analysis Report 2012[M]. Beijing: China Electric Power Press, 2012. 